\title{
Preparticipation Screening of Athletic Participant: A Proposal for the Cardiologist
}

\author{
Ebru Ipek Turkoglu, Hasan Güngör', Oktay Ergene², Antonio Pelliccia \\ ORCID: \\ Ebru Ipek Turkoglu: https://orcid.org/0000-0002-2321-8868 \\ Hasan Gungor: https://orcid.org/0000-0002-4462-3029 \\ Oktay Ergene: https://orcid.org/0000-0003-1775-4063 \\ Antonio Pelliccia: https://orcid.org/0000-0003-4174-3401
}

Department of Cardiology, Izmir Kemalpasa State Hospital, ${ }^{2}$ Department of Cardiology, School of Medicine, Dokuz Eylul University, İzmir, ${ }^{1}$ Department of Cardiology, School of Medicine, Adnan Menderes University, Aydın, Turkey, ${ }^{3}$ Coni Istituto di Medicina e Scienza dello Sport, Rome, Italy

\section{Abstract}

Hence, the sudden cardiac death (SCD) in sports activity is a rare event, it affects the community deeply. To prevent the SCD on the athletic field, screening of athletic participants is necessary. Lack of randomised controlled trials in sports-cardiology makes the decision-making process hard for the physician. Hereby, a screening model including a family and personal history, an appropriate physical examination of the athlete and 12-lead electrocardiogram, is proposed for athletic participants based on a review of current literature and in accord with the European recommendations.

Keywords: Athletic population, electrocardiogram, preparticipation screening, sudden cardiac death

\section{INTRODUCTION}

Sudden cardiac death (SCD) itself is a devastating event, especially when it occurs during an athletic activity in a previously healthy individual. Although the exact incidence of SCD is not known, $1-2$ of 100,000 athletes die suddenly in the age group between 12 and 35 years as an estimation. ${ }^{[1]}$ Although the incidence of SCD is low in the athletic population, Italian experience suggests the incidence is approximately three times greater than the sedentary counterparts (estimated at $2.3 / 100,000$ vs. $0.9 / 100,000$ individuals). However, the only exception is a recent study from Denmark that did not support these results and showed a reduced SCD incidence in the athletic population. ${ }^{[1-3]}$

Sports activity itself does not increase mortality but may act as a trigger for lethal arrhythmias in predisposed participants with underlying, even silent, cardiac disease. ${ }^{[1]}$ Because of the catastrophic effects of SCD of an apparently healthy individual, every precaution should be taken to identify the high-risk individuals and prevent SCD on the athletic field.

Received: 04-03-2020 Revised: 24-03-2020 Accepted: 13-04-2020

Published Online: 11-06-2020

\begin{tabular}{|l|l|}
\hline \multicolumn{3}{|c|}{ Access this article online } \\
\hline Quick Response Code: & Website: \\
& \\
http://www.ijcva.com
\end{tabular}

\section{BACKGROUND}

Observational evidence about the benefits of regular physical activity and sports participation outweigh by far the risk of $\mathrm{SCD},{ }^{[4]}$ and regular exercise should be encouraged in all ages.

As a result, preparticipation screening (PPS) of the athletic population has become more popular with the growing number of individuals who want to participate in sports activities as part of an active lifestyle and medico-legal issues regarding SCD on the athletic field.

Because of the lack of specific regulation in Turkey, either family physicians or cardiologists are confused about the PPS process. The present work aimed to establish a practical proposal for PPS of the athletic participants of all ages.

Address for correspondence: Dr. Ebru Ipek Turkoglu, Department of Cardiology, Izmir Kemalpasa State Hospital, İzmir 35170, Turkey. E-mail: dripek73@yahoo.com

This is an open access journal, and articles are distributed under the terms of the Creative Commons Attribution-NonCommercial-ShareAlike 4.0 License, which allows others to remix, tweak, and build upon the work non-commercially, as long as appropriate credit is given and the new creations are licensed under the identical terms.

For reprints contact: WKHLRPMedknow_reprints@wolterskluwer.com

How to cite this article: Turkoglu EI, Güngör H, Ergene O, Pelliccia A. Preparticipation screening of athletic participant: A proposal for the cardiologist. Int J Cardiovasc Acad 2020;6:35-40. 


\section{Preparticipation screening process}

The most important factor in PPS is the definition of athletic participant. Before 2016, the definition was "competitive athlete," for the person who participates in regular competition as an individual or as a part of an organized team, with regular and intense training under unique pressure to progress in achievement and performance. ${ }^{[5,6]}$

Recent observations showed the incidence of SCD is similar in both competitive and noncompetitive athletes ${ }^{[7]}$ and it is recognized that athletic participants, either recreational or amateur, usually show the behavior to exert physically up to their limits. ${ }^{[1]}$ Therefore, the definition of an athletic participant has been revised in the latest recommendation of the European Society of Cardiology (ESC). Individuals of any age, either amateur or professional, who exercise on a regular basis, independent of the competitive status, are defined as athletic participants, according to the last European guideline.

The second important issue is the cause of SCD on the field. If the causes of SCD of the athletic population are identified, the screening can be done for these and precautions to prevent SCD on the field can be taken. Age is an important determinant for the cause of SCD. Coronary artery disease $(\mathrm{CAD})$ is the leading factor of SCD in adult and older (age $>35$ years) athletes, while young athletes (age between 12 and 35 years) die mostly because of a variety of cardiac disease, including mostly genetic diseases such as hypertrophic or arrhythmogenic cardiomyopathy or congenital anomalies. The causes of SCD in young athletes are given in Table $1 .^{[1,6]}$

The most important debate is about the screening process. The aim here should be not only to prevent SCD but also to avoid unnecessary disqualification and advanced-expensive diagnostic work-up. Given the fact that the majority of older athletes die because of $\mathrm{CAD}$, some additional testing might be beneficial in high-risk older athletes. ${ }^{[8]}$

Screening protocols vary from country to country, and currently, broad-based systematic screening of athletes of any level is enforced in several countries, including the USA, Italy, and Israel. While in the USA, screening is done with only family/ personal history and appropriate physical examination, PPS in Italy and Israel is done with an electrocardiogram (ECG) on top of that. Both of European and American recommendations agreed to include a detailed familial and personal history and an appropriate physical examination because most of the common causes of SCD in the athletic population have either genetic origin or lead to clinical important sign or symptom, but there are still debates on the additional ECG screening.

Twelve-lead ECG has the potential to detect some lethal conditions such as cardiomyopathies, myocarditis, preexcitation syndromes, and channelopathies, which are responsible for up to $60 \%$ of the SCD in athletes. ${ }^{[5]}$ The addition of ECG into the PPS protocol enhances the sensitivity to detect cardiomyopathies or channelopathies from $25 \%$ to $>90 \% .{ }^{[9]}$ With the implementation of ECG into the PPS protocol, SCD in young competitive athletes decreased by approximately $90 \%$ in Italy. ${ }^{[10]}$

On the other hand, the view from the USA highlights the low incidence of SCD in this population and the issue of the false-negative and false-positive ECG results, as well as cost and resource availability. ${ }^{[11,12]}$ The main concern of these recommendations lies in balancing the detection of underlying diseases and not increasing the costs of advanced diagnostic workup with the unnecessary disqualification of athletic individuals, which brings the interpretation of athlete's ECG in a crucial position.

Consequently, for the accurate interpretation of the athlete's ECG, a big effort has been made since 2010. Regular and long-term intensive exercise causes some ECG changes in the heart, which reflects normal physiological adaptation and has to be distinguished from the abnormal findings. The first recommendation on athlete's ECG has been published in 2010 by ESC ${ }^{[13]} \mathrm{After}$ that, these criteria have been updated in Seattle to decrease the false-positive results (Seattle Criteria), and in 2016 ESC has further revised the ECG criteria. ${ }^{[1,14]}$ Because of the limits of the athletic heart can be overlapping those described as pathologic, an international group has defined the international criteria on athlete's ECG in 2017. The main difference of international criteria on athlete's ECG is to bring

\footnotetext{
Table 1: The causes of sudden cardiac death in young athletes

$\mathrm{HCM}$

AR/LVC

CCAA

Myocarditis and dilated cardiomyopathy

Premature coronary atherosclerosis

Conduction system abnormalities (ventricular preexcitation and channelopathies)

Aortic rupture (Marfan syndrome)

Mitral valve prolapse

Commotio cordis

The causes of SCD in young athletes (age between 12 and 35 years) are given in the Table 1. The ratio of the cause changes from country to country. HCM is the leading cause of SCD in USA, while AR/LVC in Italy. An anomalous origin of left coronary artery from the right aortic sinus with an interarterial course between aorta and pulmonary artery is the most common CCAA related to SCD. Although not a cardiac disease and previously thought extremely rare, commotion cordis reports are increasing especially in adolescent males with a mean age of 14 years. ${ }^{[1,5,6]} \mathrm{HCM}$ : Hypertrophic cardiomyopathy, AR/LVC: Arrhythmogenic right or left ventricular cardiomyopathy, CCAA: Congenital coronary artery anomaly, SCD: Sudden cardiac death
} 
a "borderline" definition beside physiologic and pathological features to avoid false-positive results, unnecessary diagnostic workup, and disqualification. ${ }^{[15]}$ Another important tool of daily cardiology practice, transthoracic echocardiography (TTE) is an established imaging modality of athletes to be indicated when there is a suspicion of cardiac disease on physical examination or ECG, but in the mass-screening protocol, the addition of TTE does not increase diagnostic sensitivity to identify cardiomyopathies. ${ }^{[1,16-18]}$

\section{Family and personal history}

Medical history consists of an important part of the PPS; hence, a majority of diseases related to SCD during sport are genetically inherited, mostly with an autosomal dominant pattern. In the presence of any close relative with a premature heart attack or SCD (younger than 50 ages in males and 65 ages in females), or cardiomyopathy, or Marfan, long QT, Brugada Syndromes, severe arrhythmic or coronary or other cardiac disabling disease is considered as positive family history and requires further diagnostics. Chest pain or discomfort, syncope or near-syncope, palpitation with exercise, unexplained dyspnea, or fatigue with the degree of exercise should be accepted as a positive sign, which indicates further work-up. Furthermore, any previous cardiac diagnosis or any restriction from sports should be questioned on individual bases. ${ }^{[5]}$

\section{Physical examination for preparticipation screening}

Physical examination of the athletic population for PPS should be especially focused on the causes of SCD in this population. Musculoskeletal findings for Marfan Syndrome, reduced femoral artery pulses suggestive for aortic coarctation, any clicks or abnormal heart sounds or murmurs $>2 / 6$ grade indicative for valvular abnormality are considered positive and require further diagnostics. Furthermore, irregular heart rhythm or high blood pressure should be investigated further. ${ }^{[5]}$

\section{Athlete's electrocardiogram}

Hence an appropriate evaluation of an athlete's ECG is fundamental in the PPS process including ECG, anyone interested in this area should be familiar with the ECG changes in this special population. ECG changes related to regular exercise are defined "normal," while ECG changes suggestive of cardiomyopathy should be recognized early and treated properly. There are also some ECG changes, which should not be considered pathological if they present in isolation and defined as borderline. Normal, borderline, and pathological ECG findings, according to the latest international recommendations, are defined below and summarized in Tables 2-4. ${ }^{[15]}$

\section{Normal electrocardiogram changes in athletes}

Regular, intense, and long-term exercise induces electrical and structural remodeling in the heart. The knowledge of physiological changes is crucial for all physicians taking care of the athletic populations. Isolated QRS voltage criteria for left or right ventricular hypertrophy are considered physiologic and do not require further assessment. If there is an underlying disease, additional pathological features are present. Early

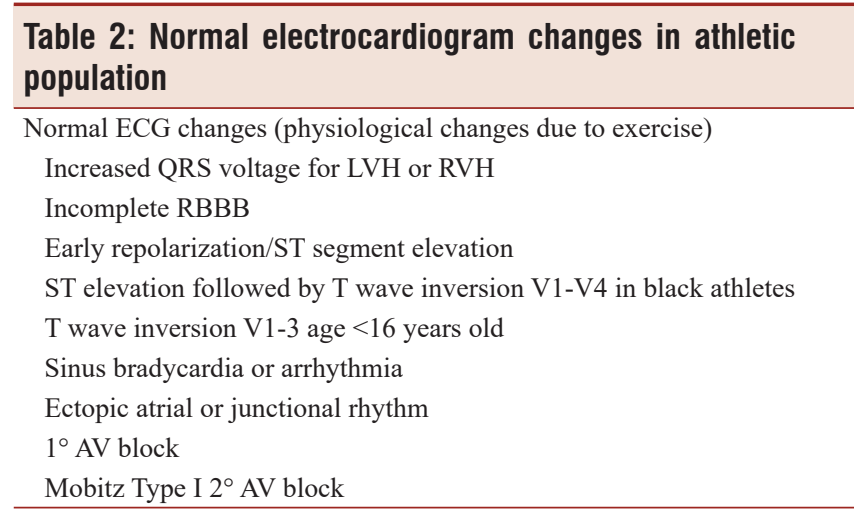

Normal ECG changes in an asymptomatic athlete with no family history do not require further diagnostic workup. ${ }^{[15]} \mathrm{LVH}$ : Left ventricular hypertrophy, RVH: Right ventricular hypertrophy, RBBB: Right bundle branch block, AV: Atrioventricular, ECG: Electrocardiogram

\section{Table 3: Pathological electrocardiogram changes in} athletic population

Pathological ECG changes (not related to exercise)
TWI
ST segment depression
Pathological Q waves
Complete LBBB
QRS $\geq 140 \mathrm{~ms}$ duration
Epsilon wave
Ventricular preexcitation
Prolonged QT interval
Brugada Type 1 pattern
Profound sinus bradycardia $<30 \mathrm{bpm}$
PR interval $\geq 400$ ms
Mobitz Type II $2^{\circ}$ AV block
$3^{\circ}$ AV block
$\geq 2$ PVCs
Atrial tachyarrhythmias
Ventricular tachyarrhythmias

Pathological ECG changes in athletic population always require further evaluation for pathological cardiovascular diseases associated with sudden cardiac death. ${ }^{[15]}$ TWI: T wave inversion, LBBB: Left bunble brunch block, PVCs: Premature ventricular contractions, ECG: Electrocardiogram, AV: Atrio-ventricular

\section{Table 4: Borderline electrocardiogram changes}

\footnotetext{
Borderline ECG changes in isolation do not require further evaluation Left axis deviation

Left atrial enlargement

Right axis deviation

Right atrial enlargement

Complete RBBB

Borderline ECG changes in isolation do not require any further evaluation, but in the presence of 2 or more findings, further evaluation is required to investigate any pathological cardiovascular disorder related to sudden cardiac death in athletes according to the International Consensus Standards for Athlete's ECG ${ }^{[15]}$ RBBB: Right bundle brunch block, ECG: Electrocardiogram
}

repolarization ( $\mathrm{J}$-point elevation $>0.1 \mathrm{mV}$ ) is common in athletes, especially in young participants, males, and of black 
ethnicity. Early repolarization with concave ST-segment elevation and peaked T-wave inversion (TWI) is seen in up to $45 \%$ of Caucasian and $63 \%-91 \%$ of black athletes. $[15,19,20] \mathrm{J}$-point elevation and convex ST-segment elevation in the anterior (V1-4) leads with TWI in black athletes are considered normal variants related to ethnicity and do not require further assessment. ${ }^{[15,20-22]}$ The juvenile ECG pattern is defined as TWI in the anterior precordial leads (V1-V3) in an adolescent athlete, who's age is between 12 and 16 years and does not require further evaluation in the absence of symptoms, signs, or familial history. ${ }^{[15]}$ The incomplete right bundle branch block (RBBB) does not require further diagnostic workup if family/personal history and physical exam are normal. ${ }^{[13]}$ The increased vagal tone in athletes is associated with some arrhythmias, such as sinus bradycardia, sinus arrhythmia, and less commonly junctional or ectopic atrial rhythms, first- and second-degree type 1 (Wenckebach) atrioventricular (AV) block. ${ }^{[15,19,23-26]}$ Recently, not only the increased vagal tone but also the electrical remodeling of the sino-atrial node is considered to cause those arrhythmias. In the absence of symptoms, the heart rates $\geq 30$ beats/minute are accepted normal, and bradycardia should resolve with the physical activity. ${ }^{[15]}$

\section{Borderline electrocardiogram changes in athletes}

The latest International Athlete's ECG recommendation has defined a borderline category to balance sensitivity (disease detection) and specificity (false-positive). Axis deviation and atrial enlargement are responsible for $>40 \%$ of abnormal ECG patterns but do not correlate with underlying cardiac disease. ${ }^{[15,27]}$

While complete $\mathrm{RBBB}$ is present in $1 \%$ of the general population, the prevalence in athletes is reported as $0.5 \%-2.5 \%{ }^{[15,28-31]}$ A study from the USA reported athletes with complete RBBB demonstrated larger right ventricular dimensions but no pathological structural cardiac disease. ${ }^{[15,32]}$ Based on the data, left or right axis deviation, left or right atrial enlargement and complete RBBB defined if identified in isolation, are considered as borderline changes and do not require further work-up. ${ }^{[15]}$

\section{Pathological electrocardiogram changes in athletes}

Pathological ECG changes in athletes are not related to the intensive exercise and always require further diagnostic workup with a temporary restriction of the athletic individual from participation.

TWI $\geq 1 \mathrm{~mm}$ in depth in 2 or more contiguous leads is abnormal, except TWI in V1-4 in black athletes and TWI in V1-3 in athletes younger than 16 years. ${ }^{[15]}$ TWI in inferior or lateral leads is common in HCM. ${ }^{[33-36]}$ Data showed TWI in the lateral or inferolateral leads in athletes is seen with the presence of cardiomyopathy. ${ }^{[15,20,37-39]}$ Similarly, an anterior TWI beyond $\mathrm{V} 2$ in a nonblack athlete older than 16 years should raise suspicion for arrhythmogenic right ventricular cardiomyopathy. ST-segment depression also is not a consequence of athletic training and should be investigated. While interpreting pathological Q-waves, lead misplacement should be taken into account. A high lead placement can result in a pseudo-septal infarct pattern, as pathological Q-waves in V1-V2. ${ }^{[15,40]}$ For asymptomatic athletes, pathological Q-waves definition has been modified as a $Q / R$ ratio $\geq 0.25$ or $\geq 40 \mathrm{~ms}$ in duration in 2 or more contiguous leads except III and aVR, to decrease false-positive results. ${ }^{[15]}$ Complete left bundle branch block is always abnormal and requires further workup. Regardless of QRS morphology, profound nonspecific intra-ventricular conduction delay (IVCD) defined as $\geq 140 \mathrm{~ms}$ is also an abnormal finding.

In an asymptomatic athlete, while a short PR interval without wide QRS or delta wave does not require further workup, Wolf-Parkinson-White pattern (PR $<120 \mathrm{~ms}$ and delta wave and QRS $>120 \mathrm{~ms}$ ) warrants further testing. ${ }^{[15]}$ Prolonged QT interval also important hence congenital long QT syndrome affects 1 in 2000 individuals. ${ }^{[41]}$ Accurate measurement of corrected QT (QTc) interval is crucial and to balance the false positive and false negative results, the QTc's cut-off is chosen for male $\geq 470$ and female $\geq 480 \mathrm{~ms}$. The exact cut-off and clinical significance of a short QT interval in the athletic population is not known. According to the latest recommendation, a QTc $<320 \mathrm{~ms}$ is considered as short QT, but it should only be investigated if there are some concerning clinical markers. ${ }^{[15]}$ Athletes with type 1 Brugada pattern should be investigated even in the absence of symptoms, but potential factors for Brugada-like ECG patterns such as hyperkalemia, fever, some medications, and lead misplacement should be taken into account. ${ }^{[15]}$

Sinus bradycardia is common in conditioned athletes, but a marked sinus bradycardia $<30$ beats/minute or a PR $\geq 400 \mathrm{~ms}$ should be investigated. High-grade AV blocks such as Type II second-degree (Mobitz type 2) or third-grade AV blocks warrant further assessment. AV dissociation without block is not pathological, but the expression of autonomic mismatch. Atrial and ventricular tachyarrhythmias require further evaluation. Multiple premature ventricular contractions (PVCs $\geq 2$ in 12 lead ECG) are considered abnormal and should be investigated for underlying disease, although they are usually benign. ${ }^{[15]}$ A recent report identified PVC according to their morphology in 12-lead ECG into common (usually benign) and uncommon (may represent the expression of underlying cardiac disease) groups and suggests using the morphology other than the number of PVC. ${ }^{[42]}$

\section{Preparticipation screening of older athletes}

Hence, the main cause of SCD in athletes older than 35 years is the CAD; screening should be done accordingly. Both European and American guidelines recommend using screening questionnaires to identify high-risk individuals. ${ }^{[8,43]}$ The aim of the questionnaire is to reveal an underlying heart disease and decrease the need for physician interaction. The questionnaire proposal for older athletes is given in Table 5. To encourage exercise and screen a bevy of people, if a low-intensity activity is aimed and self-assessment with the questionnaire went well, no further testing is recommended. If 
any the questionnaire is positive or moderate to high exercise activity is aimed, screening by a physician is recommended. Screening should be done with history, physical examination, ECG, and cardiovascular risk assessment such as SCORE risk charts. High-risk profile is summarized in Table 6. If screening is positive, then a maximal exercise testing is recommended, and according to its result, approval for exercise or further testing is determined. It is important to keep in mind here the exercise test is applied as a part of a risk stratification strategy and not simply for rule-in or rule-out CAD. ${ }^{[8]}$

\section{Conclusion}

The need for a national PPS modality is a growing issue according to the request for PPS of athletic participants such as professional athletes and athletes from either schools or sports federations or sports clubs in Turkey in line with the rest of the world. Hereby, a common national PPS model similar to the European recommendations is proposed with the hope to standardize the care for athletes of any age and help colleagues in the decision-making process.

A family and personal history, appropriate physical examination, and ECG are recommended in PPS of young athletes. For older athletes, an additional risk assessment is needed. If the athlete

\section{Table 5: Questionnaire for older athletes}

Has a doctor ever said that you have a heart disease and recommended only medically supervised activity? (Did you ever have a heart attack, coronary angiogram, coronary stent or heart surgery?)

Yes/no

Has a doctor ever recommended you medication for heart disease or hypertension?

Yes/no

Do you ever have chest pain or palpitation with physical activity?

Yes/no

Do you ever have dizziness, fainting, blackouts or unreasonable shortness of breath?

Yes/no

Are you aware, through your own experience or a doctor's advise, of any other reason that would you prohibit from you exercising?

Yes/no

Proposed questionnaire for older athletes. If all the answers are no, there is no need for further work-up in individuals aiming low intensity activity. Adopted from Ballady. Circulation 1998;97:2283-93

\section{Table 6: High risk profile for older athletes}

The presence of multiple risk factors with an estimated 10 -year risk $>5 \%$ according to score risk chart

Significant high blood cholesterol (LDL $>240 \mathrm{mg} / \mathrm{dl}$ ) or blood pressure (>180/110 mmHg)

Diabetes mellitus with microalbuminia

Individuals with a strong family history for premature CVD in firstdegree relative younger than 50 years or individuals with a BMI more than 28

High risk profile for older adults. In the presence of even 1 factor maximal exercise testing is recommended for risk stratification. ${ }^{[8]}$ CVD: Cardiovascular disease, BMI: Body mass index, LDL: Low-density lipoprotein has high-risk profile, an exercise test is recommended as part of risk stratification. It is important to keep in mind and share the knowledge with the athletic participants, that the risk of SCD (1-2/100,000) still remains despite all the screening process, but the risk is very low, and the beneficial effects of exercise are overwhelming.

\section{Financial support and sponsorship \\ Nil.}

\section{Conflicts of interest}

There are no conflicts of interest.

\section{References}

1. Mont L, Pelliccia A, Sharma S, Biffi A, Borjesson M, Terradellas JB, et al. Pre-participation cardiovascular evaluation for athletic participants to prevent sudden death: Position paper from the EHRA and the EACPR, branches of the ESC. Endorsed by APHRS, HRS, and SOLAECE. Europace 2017;19:139-63.

2. Corrado D, Basso C, Rizzoli G, Schiavon M, Thiene G. Does sports activity enhance the risk of sudden death in adolescents and young adults? J Am Coll Cardiol 2003;42:1959-63.

3. Holst AG, Winkel BG, Theilade J, Kristensen IB, Thomsen JL, Ottesen GL, et al. Incidence and etiology of sports-related sudden cardiac death in Denmark--implications for preparticipation screening. Heart Rhythm 2010;7:1365-71.

4. Borjesson M, Dellborg M, Niebauer J, LaGerche A, Schmied C, Solberg EE, et al. Recommendations for participation in leisure time or competitive sports in athletes-patients with coronary artery disease: A position statement from the Sports Cardiology Section of the European Association of Preventive Cardiology (EAPC). Eur Heart J 2019;40:13-8.

5. Corrado D, Pelliccia A, Bjørnstad HH, Vanhees L, Biffi A, Borjesson M, et al. Cardiovascular pre-participation screening of young competitive athletes for prevention of sudden death: proposal for a common European protocol. Consensus Statement of the Study Group of Sport Cardiology of the Working Group of Cardiac Rehabilitation and Exercise Physiology and the Working Group of Myocardial and Pericardial Diseases of the European Society of Cardiology. Eur Heart J 2005;26:516-24.

6. Maroon BJ, Zipes DF, Kovacs RJ. Eligibility and disqualification recommendations for competitive athletes with cardiovascular abnormalities: Preamble, principles, and general considerations. A scientific statement from the American Heart Association and American College of Cardiology. J Am Coll Cardiol 2015;66:2343-49.

7. Risgaard B, Winkel BG, Jabbari R, Glinge C, Ingemann-Hansen $\mathrm{O}$, Thomsen JL, et al. Sports-related sudden cardiac death in a competitive and a noncompetitive athlete population aged 12 to 49 years: Data from an unselected nationwide study in Denmark. Heart Rhythm 2014;11:1673-81.

8. Borjesson M, Urhausen A, Kouidi E, Dugmore D, Sharma S, Halle M, et al. Cardiovascular evaluation of middle-aged/senior individuals engaged in leisure-time sport activities: Position stand from the sections of exercise physiology and sports cardiology of the European Association of Cardiovascular Prevention and Rehabilitation. Eur J Cardiovasc Prev Rehabil 2011;18:446-58.

9. Harmon KG, Zigman M, Drezner JA. The effectiveness of screening history, physical exam, and ECG to detect potentially lethal cardiac disorders in athletes: a systematic review/meta-analysis. J Electrocardiol 2015;48:329-38.

10. Corrado D, Basso C, Pavei A, Michieli P, Schiavon M, Thiene G. Trends in sudden cardiovascular death in young competitive athletes after implementation of a pre-participation screening program. JAMA 2006;296:1593-601.

11. Maroon BJ, Levine BD, Kovacs RJ, Washington RL, Maroon MS, Baggish AL. Eligibility and disqualification recommendations for competitive athletes with cardiovascular abnormalities: Task force 2: 
Preparticipation screening for Cardiovascular Disease in Competitive Athletes. A Scientific Statement from the American Heart Association and American College of Cardiology. JACC 2015:66:2356-61.

12. Maron BJ, Haas TS, Doerer JJ, Thompson PD, Hodges JS. Comparison of U.S. and Italian experiences with sudden cardiac deaths in young competitive athletes and implications for preparticipation screening strategies. Am J Cardiol. 2009;104:276-80.

13. Corrado D, Pelliccia A, Heidbuchel H, Sharma S, Link M, Basso C, et al. On behalf of the Sections of Sports Cardiology of the European Association of Cardiovascular Prevention and Rehabilitation; and the Working Group of Myocardial and Pericardial Disease of the European Society of Cardiology. Recommendations for interpretation of 12-lead electrocardiogram in the athlete. Eur Heart J 2010;31:243-59.

14. Drezner JA, Ackerman MJ, Anderson J, Ashley E, Asplund CA, Baggish AL, et al. Electrocardiographic interpretation in athletes: The 'Seattle Criteria'. Sports Med 2013;47:122-4.

15. Sharma S, Drezner JA, Baggish A, Papadakis M, Wilson MG, Prutkin JM, et al. International recommendations for electrocardiographic interpretation in athletes. J Am Coll Cardiol 2017;69:1057-75.

16. Vetter VL, Dugan N, Guo R, Mercer-Rosa L, Gleason M, Cohen M, et al. A pilot study of the feasibility of heart screening for sudden cardiac arrest in healthy children. Am Heart J 2011;161:1000-6.e3.

17. Anderson JB, Grenier M, Edwards NM, Madsen NL, Czosek RJ, Spar DS, et al. Usefulness of combined history, physical examination, electrocardiogram, and limited echocardiogram in screening adolescent athletes for risk for sudden cardiac death. Am J Cardiol 2014;114:1763-7.

18. Riding NR, Sharma S, Salah O, Khalil N, Carré F, George KP, et al. Systematic echocardiography is not efficacious when screening an ethnically diverse cohort of athletes in West Asia. Eur J Prev Cardiol 2015;22:263-70

19. Papadakis M, Basavarajaiah S, Rawlins J, Edwards C, Makan J, Firoozi S, et al. Prevalence and significance of T-wave inversions in predominantly Caucasian adolescent athletes. Eur Heart J 2009;30:1728-35.

20. Papadakis M, Carre F, Kervio G, Rawlins J, Panoulas VF, Chandra N, et al. The prevalence, distribution, and clinical outcomes of electrocardiographic repolarization patterns in male athletes of African/ Afro-Caribbean origin. Eur Heart J 2011;32:2304-13.

21. Sheikh N, Papadakis M, Carre F, Kervio G, Panoulas VF, Ghani S, et al. Cardiac adaptation to exercise in adolescent athletes of African ethnicity: An emergent elite athletic population. Br J Sports Med 2013;47:585-92.

22. Di Paolo FM, Schmied C, Zerguini YA, Junge A, Quattrini F, Culasso F, et al. The athlete's heart in adolescent Africans: An electrocardiographic and echocardiographic study. J Am Coll Cardiol 2012;59:1029-36.

23. Rawlins J, Carre F, Kervio G, Papadakis M, Chandra N, Edwards C, et al. Ethnic differences in physiological cardiac adaptation to intense physical exercise in highly trained female athletes. Circulation 2010;121:1078-85.

24. Sharma S, Whyte G, Elliott P, Padula M, Kaushal R, Mahon N, et al. Electrocardiographic changes in 1000 highly trained junior elite athletes. Br J Sports Med 1999;33:319-24.

25. Stein R, Medeiros CM, Rosito GA, Zimerman LI, Ribeiro JP. Intrinsic sinus and atrioventricular node electrophysiologic adaptations in endurance athletes. J Am Coll Cardiol 2002;39:1033-8.

26. Northcote RJ, Canning GP, Ballantyne D. Electrocardiographic findings in male veteran endurance athletes. Br Heart J 1989;61:155-60.

27. Gati S, Sheikh N, Ghani S, Zaidi A, Wilson M, Raju H, et al. Should axis deviation or atrial enlargement be categorised as abnormal in young athletes? The athlete's electrocardiogram: Time for re-appraisal of markers of pathology. Eur Heart J 2013;34:3641-8.

28. Pelliccia A, Culasso F, Di Paolo FM, Accettura D, Cantore R, Castagna W, et al. Prevalence of abnormal electrocardiograms in a large, unselected population undergoing pre-participation cardiovascular screening. Eur Heart J 2007;28:2006-10.

29. Fudge J, Harmon KG, Owens DS, Prutkin JM, Salerno JC, Asif IM, et al. Cardiovascular screening in adolescents and young adults: A prospective study comparing the Pre-participation Physical Evaluation Monograph $4^{\text {th }}$ Edition and ECG. Br J Sports Med 2014;48:1172-8.

30. Magalski A, McCoy M, Zabel M, Magee LM, Goeke J, Main ML, et al. Cardiovascular screening with electrocardiography and echocardiography in collegiate athletes. Am J Med 2011;124:511-8.

31. Baggish AL, Hutter AM Jr, Wang F, Yared K, Weiner RB, Kupperman E, et al. Cardiovascular screening in college athletes with and without electrocardiography: A cross-sectional study. Ann Intern Med 2010;152:269-75.

32. Kim JH, Noseworthy PA, McCarty D, Yared K, Weiner R, Wang F, et al. Significance of electrocardiographic right bundle branch block in trained athletes. Am J Cardiol 2011;107:1083-9.

33. Rowin EJ, Maron BJ, Appelbaum E, Link MS, Gibson CM, Lesser JR, et al. Significance of false negative electrocardiograms in preparticipation screening of athletes for hypertrophic cardiomyopathy. Am J Cardiol 2012;110:1027-32.

34. Chen X, Zhao T, Lu M, Yin G, Xiangli W, Jiang S, et al. The relationship between electrocardiographic changes and CMR features in asymptomatic or mildly symptomatic patients with hypertrophic cardiomyopathy. Int J Cardiovase Imaging 2014;30 Suppl 1:55-63.

35. Sheikh N, Papadakis M, Schnell F, Panoulas V, Malhotra A, Wilson M, et al. Clinical Profile of Athletes With Hypertrophic Cardiomyopathy. Circ Cardiovasc Imaging 2015;8:e003454.

36. BentRE, WheelerMT, Hadley D, Knowles JW, PavlovicA, Finocchiaro G, et al. Systematic Comparison of Digital Electrocardiograms From Healthy Athletes and Patients With Hypertrophic Cardiomyopathy. J Am Coll Cardiol 2015;65:2462-3.

37. Pelliccia A, Di Paolo FM, Quattrini FM, Basso C, Culasso F, Popoli G, et al. Outcomes in athletes with marked ECG repolarization abnormalities. N Engl J Med 2008;358:152-61.

38. Schnell F, Riding N, O'Hanlon R, Axel Lentz P, Donal E, Kervio G, et al. Recognition and significance of pathological T-wave inversions in athletes. Circulation 2015;131:165-73.

39. Chandra N, Bastiaenen R, Papadakis M, Panoulas VF, Ghani S, Duschl J, et al. Prevalence of electrocardiographic anomalies in young individuals: Relevance to a nationwide cardiac screening program. J Am Coll Cardiol 2014;63:2028-34.

40. MacAlpin RN. Clinical significance of QS complexes in V1 and V2 without other electrocardiographic abnormality. Ann Noninvasive Electrocardiol 2004;9:39-47.

41. Schwartz PJ, Stramba-Badiale M, Crotti L, Pedrazzini M, Besana A, Bosi G, et al. Prevalence of the congenital long-QT syndrome. Circulation 2009; 120:1761-7.

42. Corrado D, Drezner JA, D'Ascenzi F, Zorzi A. How to evaluate premature ventricular beats in the athlete: Critical review and proposal of a diagnostic algorithm Sports Med 2019:1-8. [doi: 10.1136/ bjsports-2018-100529].

43. Balady GJ, Chaitman B, Driscoll D, Foster C, Froelicher E, Gordon N, et al. AHA/ACSM scientific statement: Recommendations for cardiovascular screening, staffing, and Emergency policies at health/ fitness facilities. Circulation 1998;97:2283-93. 